\title{
Arterial Therapies of Non-Colorectal Liver Metastases
}

\author{
Gilbert Puippe $^{\mathrm{a}} \quad$ Thomas Pfammatter $^{\mathrm{a}} \quad$ Niklaus Schaefer $^{\mathrm{b}}$ \\ ${ }^{a}$ Department of Diagnostic and Interventional Radiology, University Hospital Zurich, Zurich, Switzerland, \\ ${ }^{\mathrm{b}}$ Division of Nuclear Medicine, Lausanne University Hospital, Lausanne, Switzerland
}

\section{Keywords}

Liver - Intra-arterial therapy · Embolization .

Breast cancer · Neuroendocrine · Melanoma

\section{Summary}

Background: The unique situation of the liver with arterial and venous blood supply and the dependency of the tumor on the arterial blood flow make this organ an ideal target for intrahepatic catheter-based therapies. Main forms of treatment are classical bland embolization (TAE) cutting the blood flow to the tumors, chemoembolization (TACE) inducing high chemotherapy concentration in tumors, and radioembolization (TARE) without embolizing effect but very high local radiation. These different forms of therapies are used in different centers with different protocols. This overview summarizes the different forms of treatment, their indications and protocols, possible side effects, and available data in patients with non-colorectal liver tumors. Methods: A research in PubMed was performed. Mainly clinical controlled trials were reviewed. The search terms were 'embolization liver', 'TAE', 'chemoembolization liver', 'TACE', 'radioembolization liver', and 'TARE' as well as 'chemosaturation' and 'TACP' in the indications 'breast cancer', 'neuroendocrine', and 'melanoma'. All reported studies were analyzed for impact and reported according to their clinical relevance. Results: The main search criteria revealed the following results: 'embolization liver + breast cancer', 122 results, subgroup clinical trials 16; 'chemoembolization liver + breast cancer', 62 results, subgroup clinical trials 11; 'radioembolization liver + breast cancer', 37 results, subgroup clinical trials 3 ; 'embolization liver + neuroendocrine', 283 results, subgroup clinical trials 20; 'chemoembolization liver + neuroendocrine', 202 results, subgroup clinical trials 9; 'radioembolization liver + neuroendocrine', 64 results, subgroup clinical trials 9; 'embolization liver + melanoma', 79 results, subgroup clini- cal trials 15; 'chemoembolization liver + melanoma', 60 results, subgroup clinical trials 14; 'radioembolization liver + melanoma', 18 results, subgroup clinical trials 3. The term 'chemosaturation liver' was tested without indication since only few publications exist and provided us with five results and only one clinical trial. Conclusion: Despite many years of clinical use and documented efficacy on intra-arterial treatments of the liver, there are still only a few prospective multicenter trials with many different protocols. To guarantee the future use of these efficacious therapies, especially in the light of many systemic or surgical therapies in the treatment of non-colorectal liver metastases, further large randomized trials and transparent guidelines need to be established.

(c) 2015 S. Karger GmbH, Freiburg

\section{Introduction}

Arterial therapies of liver malignancy are commonly used techniques employing catheter systems in the hepatic arterial route. They are based on the existence of arterial tumoral hypervascularization of macroscopic lesions which draw most of their blood supply from the hepatic artery whereas normal liver parenchyma is mostly perfused by the portal venous system. The first reports of successful intra-arterial liver treatment were published in the mid1970s [1]. Interestingly, early reports already mentioned the use of intra-arterial chemotherapy or radioactive isotopes to treat malignant disease [2]. In these early studies symptom control and relief was reported in up to $65 \%$ of the patients. With the introduction of modern, specialized interventional units, micro-catheters, and subspecialized physicians the field advanced to a major player in the treatment of liver-dominant disease.

This overview describes the various intra-arterial treatment options of non-colorectal liver metastases, focusing on the use of

\section{KARGER \\ Fax +497614520714

() 2015 S. Karger GmbH, Freiburg

$1662-6664 / 15 / 0316-0414 \$ 39.50 / 0$
Prof. Niklaus Schaefer, MD

Division of Nuclear Medicine, Bureau BH/10/913 University Hospital of Lausanne

Rue du Bugnon 46, 1011 Lausanne, Switzerland niklaus.schaefer@ chuv.ch 
Fig. 1. 54-year-old man with symptomatic liver metastases of a well-differentiated neuroendocrine tumor of the small bowel. a Superselective digital subtraction angiography over a $2.7 \mathrm{~F}$ microcatheter (white arrow) of an aberrant hepatic artery to the liver segments IV and V shows two hypervascular tumors in these segments (black arrows). b Plain $\mathrm{X}$-ray after transarterial bland embolization with $40 \mu \mathrm{m}$ microspheres (Embozene ${ }^{\circledR}$; Celonova Biosciences, San Antonio, TX, USA) shows complete stasis in the embolized tumors.
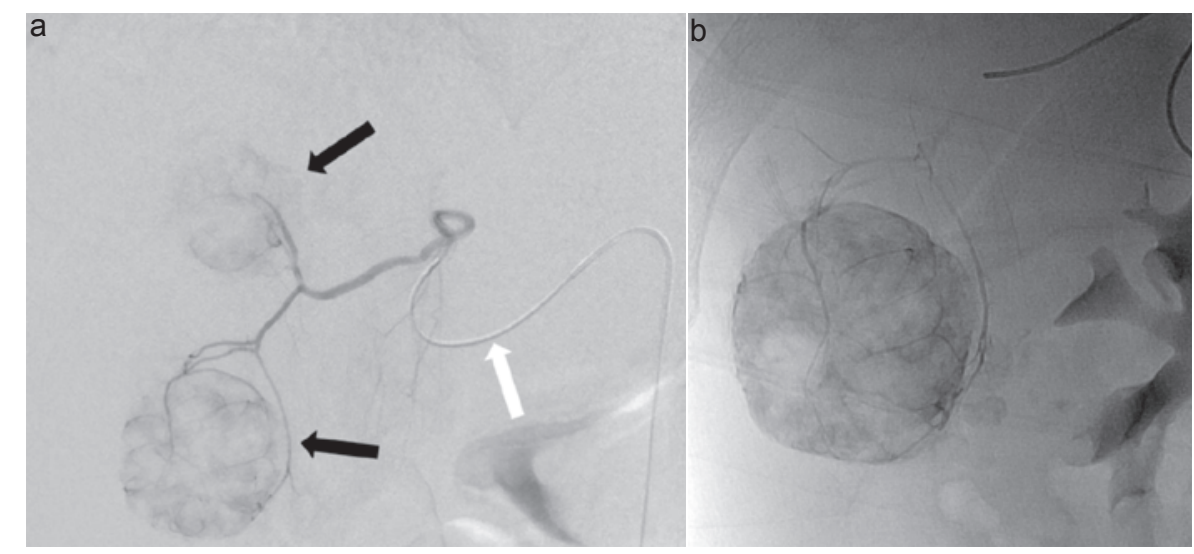

bland transarterial embolization (TAE), transarterial chemoembolization (TACE), transarterial radioembolization (TARE), and transarterial chemoperfusion (TACP) in different tumor entities.

\section{Technique of Transarterial Embolization}

\section{Definition of TAE}

TAE or bland embolization of intrahepatic arteries aims at reversible or irreversible blocking of hepatic arterial flow by using different embolic agents (e.g. polyvinyl alcohol (PVA) particles, spheres, or gelatin sponge). The pure mechanism of action is tumor ischemia. The dual blood supply of the liver provided by the hepatic artery and the portal venous system allows for blocking of the two blood systems without causing necrosis of the liver parenchyma. The target of TAE is the arterial blood supply of primary or metastatic tumors of the liver, and therefore tumors with predominant arterial blood supply seem amenable for TAE.

A variety of different embolic materials is currently available on the market. Gelfoam, a gelatin sponge, is a widely used embolic material commercially available as powder or as sheet of which small cubes or pledgets can be cut. However, the size of the pletgets is highly variable, and this may lead to a too proximal occlusion. Moreover, it is biodegradable and absorbed within 1-3 weeks; thus, it is regarded as temporary embolic material.

PVA particles are non-spherical particles of irregular shape and size which were mixed with diluted contrast media. PVA particles are seen as a permanent embolic material. Potential disadvantages are that they tend to aggregate which may also lead to too proximal embolization.

Spheres basically consist of an inert, elastic polymer that is covered with a hydrophilic surface. The shape is spherical, and calibration of sphere size is possible within very narrow ranges. Currently, a wide range of spheres are available from different manufactures, with sizes ranging from 40 to $1200 \mu \mathrm{m}$. The shape and coating make clumping less a problem than compared to non-spherical PVA. This allows for the spheres to reach the very distal capillaries of the tumor bed, resulting in a more profound ischemia. Figure 1 illustrates a bland embolization of one metastasis of a neuroendocrine tumor (NET) with $40 \mu \mathrm{m}$ microspheres.

TAE is generally used for targeted embolization, i.e., the tumorfeeding artery is selectively catheterized and embolized to avoid tissue damage of the normal liver parenchyma.

\section{Technique of Transarterial Chemoembolization}

\section{Definitions and Indications of TACE}

TACE is a local catheter-based and minimally invasive therapeutic option for patients with unresectable and liver-dominant hepatic malignancies. By definition, TACE consists of a chemotherapeutic agent mixed with an embolic material, which is then selectively administered through the feeding arteries of the tumors. This combination allows for high intratumor drug concentrations, and the occlusion of the tumor-feeding arteries causes ischemic damage and necrosis of the tumor. Therefore, TACE seems amenable in primary and secondary liver tumors, which derive their blood supply mainly from the hepatic artery (e.g. hepatocellular carcinoma (HCC), breast cancer liver metastases (BCLM), renal cell carcinoma, NET, melanoma, and bronchial carcinoma).

TACE is not uniformly defined in the literature. Different chemotherapeutic agents (e.g. doxorubicin, irinotecan, mitomycin-C, and cisplatin) as well as different embolic materials (iodized oil, gelatin sponge, PVA particles, spheres, and drug-eluting beads) are used. The conventional TACE (cTACE) consists of an injection of an emulsion of doxorubicin (usually $50-100 \mathrm{mg} / \mathrm{m}^{2}$ ) mixed with ethiodized oil (Lipiodol, Guerbet GmbH, Sulzbach, Germany), an iodinated ester derived from poppy-seed oil, followed by the administration of embolic agents (e.g. gelatin sponge particles, PVA particles, or calibrated spheres) to achieve stasis in the target vessel [3].

With the introduction of drug-eluting beads (DC Beads, Biocompatibles, Farnham, UK) a new drug delivery and embolization system became available. Drug-eluting beads are non-resorbable hydrogel beads, available in different sizes (e.g. 100-300 $\mu \mathrm{m}, 300-$ $500 \mu \mathrm{m}$ ), that can be preloaded with chemotherapeutic agents, e.g. doxorubicin (DEBDOX TACE) or irinotecan (DEBIRI TACE). 
Preclinical data of drug-eluting beads showed higher intratumor drug concentrations, a more sustained drug release into the tumor, and a low release of doxorubicin into the systemic circulation compared to cTACE [4]. Results from the PRECISION V trial comparing doxorubicin-loaded DC Beads with cTACE in patients with BCLC stage A/B HCC showed a trend towards improved tumor control, a significant reduction in serious liver toxicity, and a significantly lower rate of doxorubicin-related side effects in the DC Bead group [3].

\section{Pretreatment Considerations and Contraindications}

All patients should obtain some cross-sectional imaging either with magnetic resonance imaging, computed tomography (CT), or positron emission tomography (PET)/CT to prove liver-dominant disease and to ensure that the portal venous system is patent. The latter is important due to the risk of necrosis and liver failure following arterial embolization in patients with portal venous thrombosis. Moreover, all patients should have laboratory tests including complete blood count, liver and kidney function tests, and coagulation state [5]. It is known that a serum bilirubin level $>34 \mu \mathrm{mol} / \mathrm{l}$, $>50 \%$ liver tumor involvement, and AST levels $>100$ IU/l are associated with increased mortality following TACE [6].

\section{Intervention, Technical Requirements, and Considerations}

TACE is performed in dedicated angiography suites with local anesthesia at the puncture site. Moderate conscious sedation is rarely required. Antibiotic prophylaxis is not generally recommended but should be considered in patients with bilioenteric anastomosis, biliary stents, or incompetent sphincter of Oddi [7]. A percutaneous retrograde femoral access with insertion of a 5-6 French vascular sheath is most common. Due to the high prevalence of variant hepatic arterial anatomy of $12-49 \%$ [8], diagnostic angiography of the coeliac trunk and superior mesenteric artery should always be obtained at the time of first TACE. Filming should also include the portal venous phase to prove patency and hepatopetal flow of the portal venous system as well as to exclude collateral pathways. The mixture of chemotherapeutic agent and embolic material can be delivered in a selective, segmental, or lobar manner depending on number, size, and distribution of the tumors. The use of microcatheters allows for very selective catheterization of tumor vessels and helps to avoid vasospasms, which may hamper drug delivery.

\section{Complications Associated with TACE}

Complications following TACE can occur in up to $10 \%$. Postembolization syndrome (fever, nausea, pain) is most frequent (4.6\%) but not necessarily classified as a complication [5]. Liver failure is seen in up to $2.3 \%$. Patients with biliary stents or bilioen- teric anastomosis are at higher risk $(0-15 \%)$ for postprocedural abscess than patients with functional sphincter of Oddi (1-2\%). Inadvertent drug delivery to the gallbladder or gastrointestinal tract can cause cholecystitis $(<1 \%)$ and gastrointestinal ulcers $(<1 \%)$ [5]. Systemic side effects like skin discoloration (1.9-2.2\%), mucositis (4.3-5.6\%), and bone marrow suppression (5.4-5.6\%) are relatively rare. Alopecia is almost absent $(1.1 \%)$ with DEBDOX TACE though very frequent in patients who undergo cTACE (20.4\%) [3].

\section{Technique of Transcatheter Arterial Radio- embolization}

\section{Definitions and Indications of TARE}

The principle of TARE is strongly associated with TACE. The main difference to TACE is the choice of local radiation instead of chemotherapy to achieve tumor control. High local radiation is delivered by microspheres containing or carrying Yttrium-90 (Y90, $\mathrm{T} 1 / 264 \mathrm{~h}$ ). Y90 is a pure beta emitter with high local energies depositing local radiation over a few millimeters up to 1,000 Gray in one session. Since the distance of radiation is very short, the radiation burden of the normal liver is considered to be lower than 35 Gray. In Switzerland, the treatment is used consequently in an outpatient setting. However, since effects of radiation and chemotherapy are biologically complementary, patient selection, patient information, technical intervention, and side effects are substantially different.

In every cancer treatment, selection of patients is most important. In Switzerland, TARE is paid for patients with liver-dominant, unresectable, chemorefractory liver metastases or HCC not amenable for TACE. Most often, indications of non-colorectal metastases are breast cancer, NET/carcinomas, and melanomas, in addition to rare indications such as pancreatic cancer or sarcomas (the data for these indications will be presented in the course of this article).

However, as a result of the most recent studies, the situation might change in the near future. In our daily routine, patients after several lines of chemotherapy are the most often indications for TARE. Since many of these patients already suffer from side effects of multiple preexisting chemotherapies, meticulous choice regarding the ECOG score, liver function, and extrahepatic spread is relevant. As many of these patients are heavily pretreated, all our patients are primarily seen by an oncologist and then presented at the local tumor board. Most important factors are sufficient liver function, absence of ascites, and only moderately elevated bilirubin $(<1.5$ upper limit (UL)) and transaminases $(<5 \mathrm{UL})$. Indications and contraindications are summarized in table 1.

\section{Intervention, Technical Requirements, and Considerations}

After having received written informed consent of the patient, the first angiography of TARE preparation is performed. The pri- 
Table 1. Indication and contraindications for TARE; official status in Switzerland KLV Addendum 9.3. from July 1, 2010

\section{Indication}

Inoperable, chemotherapy-refractory liver tumors in which no other local ablative or embolizing therapy is indicated or has been non-effective

Indication through interdisciplinary hepatobiliary center with specialized surgery, interventional radiology

Signed patient consent form

Contraindications

Palpable ascites or other clinical signs of liver failure

Significant laboratory signs of liver failure (ALT, AST $>5 \times$ ULN), bilirubin $>$ 1.5 ULN

Relevant hepato-pulmonary shunt (generally $>20 \%$ )

Relevant, non-correctable reflux in stomach, duodenum, pancreas, or other intestinal organs

Dominant extrahepatic disease

mary angiography is used to plan the therapy as well as to identify and eventually coil aberrant vessels, e.g. gastric or duodenal arteries. Before removing the catheter, a total of $180 \mathrm{MBq}$ of Tc-99m microaggregated albumin (MAA) is infused to simulate the final therapeutic procedure. Patients are then transferred to the nuclear medicine department for whole-body single-photon emission computed tomography (SPECT) to predict lung shunting (e.g. in cirrhotic livers). A liver/lung shunt $>20 \%$ is considered a contraindication for TARE. However, free Tc-99m, e.g. in the ear/nose/ throat region or diffusely in the stomach, has to be considered as false-positive. There are several publications showing that the distribution of MAA in SPECT might predict the response and outcome of TARE. However, data from our group showed that perfusion CT prior to radioembolization predicts the overall survival (OS) of our patients [9]. Only after the planning procedure the selective internal radiation therapy (SIRT) might be initiated. The procedure is shown in figure 2 .

TARE can be performed using two different products, i.e. resin microspheres (Sirtex Medical Limited, North Sydney, Australia) or glass microspheres (BTG International Ltd, London, UK). Both products underlie the same mechanism of high local radiation via intra-arterial placement in the liver. The products differ in size, number of particles, radiation per particle, and variants of the administration procedure. The dose is calculated via different models by either considering body surface area (BSA model) or liver volumetry or via dosimetric modelling. In large multicenter studies, mostly easy-to-use models are applied and must be used accordingly in the respective setting (e.g. SIRFLOX study, adapted BSA model). TARE per se is performed in our institution in an outpatient setting. Exceptions are large tumors or functional NET where the systemic response due to release of cytokines and hormones can be dramatic. The procedure has to be performed in specifically labelled angiography rooms which have to be cleared after the intervention by the radiation protection staff. After the procedure, patients receive the 'Bremsstrahlung Scan' which allows the depiction of local radiation. Alternatively, time-of-flight PET can also identify high local radiation over internal pair positron emission.
Patients are seen 4 weeks after SIRT in an outpatient setting. Imaging is not recommended earlier than 3 months after radiation. However, one must be aware that interpretation of imaging might be complicated after radiation and inflammation.

\section{Complications Associated with TARE}

TARE is a safe procedure in experienced hands. However, since many new treatment centers are founded in the light of rising evidence, one must be aware of possible side effects. Most current side effects arise from systemic response to inflammation. Patients suffer from fever, swelling, tiredness, and sometimes prolonged pain. Nevertheless, most of these side effects are of short duration, i.e. only a few days. Dangerous side effects might arise from misplacement of the microspheres. Especially deposits of small amounts of spheres in the duodenum or in the gastric wall might produce long-lasting and painful ulceration or even perforation. This complication has to be avoided at all circumstances by meticulous coiling of all arteries at risk and exact catheter placement. A further dangerous complication is radiation-induced liver disease. This complication arises around 4 weeks after the therapy procedure and is primarily of clinical nature, mimicking a hepatic veno-occlusive disease. This is a very rare but potentially life-threatening complication which must be treated in close collaboration with the hepatologists.

\section{Technique of Transarterial Chemoperfusion/ Chemosaturation}

\section{Definitions and Indications of TACP}

TACP is identical to other intra-arterial treatments benefiting from differential blood supply of liver metastases and normal liver tissue. The main difference is the continuous infusion of isolated chemotherapy (e.g. melphalan). This system allows a very high dose of local chemotherapy (chemosaturation) by means of subsequent chemo-isolation through a double-balloon catheter in the venous hepatic outflow.

This rather difficult procedure has only few indications and has to our knowledge only been tested in a series of uveal melanoma.

\section{Intervention, Technical Requirements, and Considerations}

The procedure is usually carried out under general anesthesia, and full anticoagulation is needed during the whole procedure. High doses of a chemotherapy drug are then infused directly into the liver via the hepatic artery infusion catheter over a period of approximately $30 \mathrm{~min}$. During this time, blood leaving the liver passes through an extracorporeal filtration system that removes most of the chemotherapy drug before the blood is returned to the circulation via a catheter in the internal jugular vein. Since the 
Fig. 2. Overview of the diagnostic workup of a 74-year-old male with liver metastases in the right liver lobe of a high-grade gastric neuroendocrine carcinoma prior to TARE. a Digital subtraction angiography of coeliac trunk showing the large hypervascular tumor in the right lobe (black arrowheads) as well as a smaller tumor (white arrowheads) in segment VI. The gastroduodenal artery (black arrow) is patent. The right gastric artery was ligated during gastrectomy and cannot be appreciated on angiography any more. b Stain ing of the tumor continues to the portal venous phase. c Following coil embolization of the gastroduodenal artery (white arrow), the microcatheter was placed in the proper hepatic artery and 180 $\mathrm{MBq}$ of Tc-99m MAA were administered through the microcatheter. d Whole-body SPECT was acquired following Tc-99m MAA infusion. The calculated liver/lung shunt was 0.14. e Fused coronal image of SPECT showing Tc-99m MAA deposits in the liver metastases.

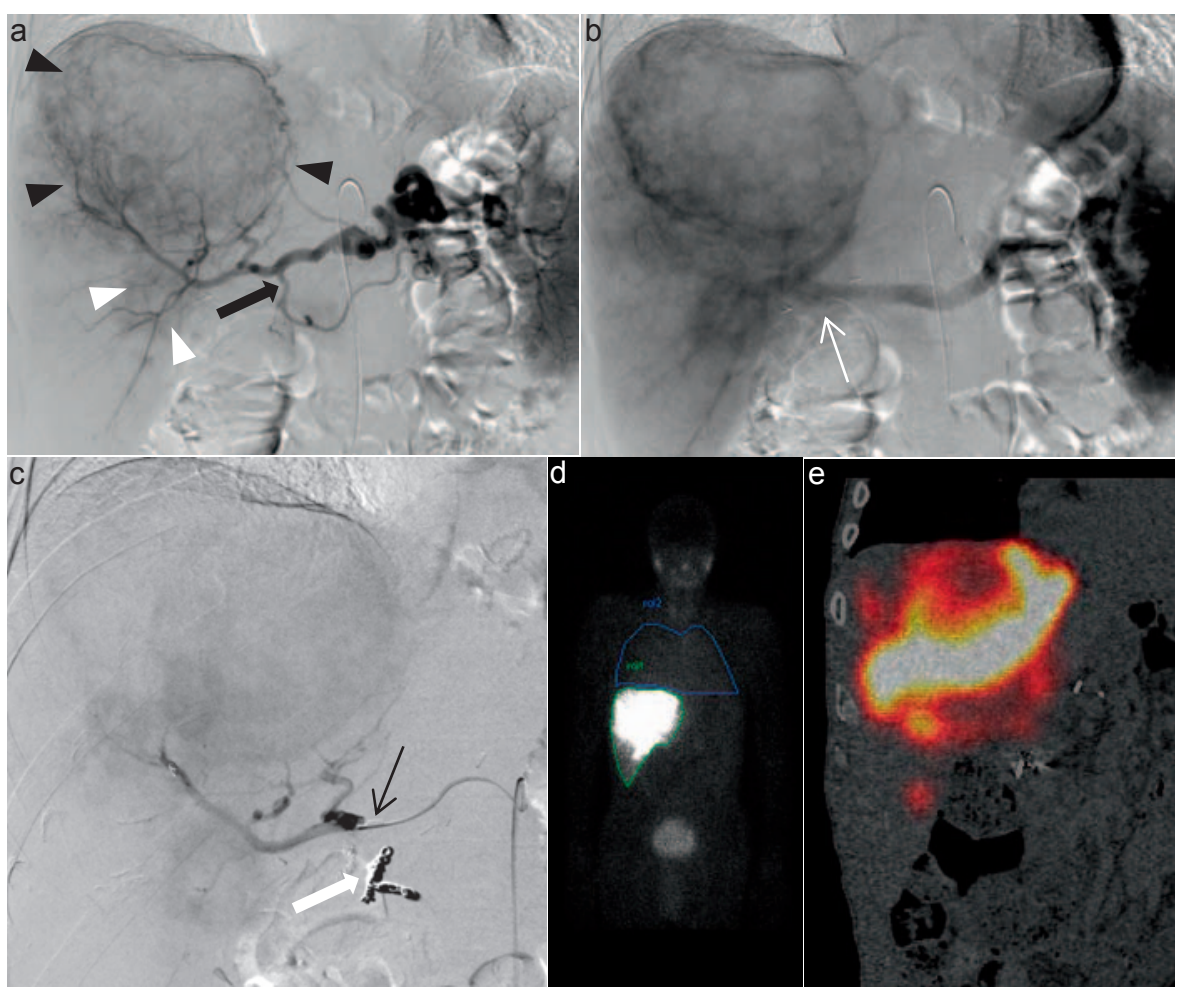

hemodynamic status changes during the procedure, an anesthetic team and a perfusion scientist are required for management.

\section{Complications Associated with TACP}

Adverse events resulting in death were reported in $4 \%$ in a study of 121 (5/121) patients, while serious neutropenia and thrombocytopenia were reported in $59 \%$ and in $80 \%$, respectively, in the same study [9]. Furthermore, 17\% had cardiovascular events (13 at the time of the procedure), and gastrointestinal events were reported in $11 \%$ (including gastritis, ulceration, perforation, bleeding, and gallbladder-related events). Moreover, patients may suffer from hemorrhage or hepatic events, making this procedure overall rather toxic.

\section{Results}

\section{Breast Cancer Liver Metastases}

BCLM are seen in up to $50 \%$ of all patients with metastatic breast cancer, and the presence of liver metastases is reported to be associated with poor outcome, with median survival rates of less than 6 months [10]. Newer epidemiological data show that median survivals of up to 16.3 months can be achieved with modern adjuvant chemotherapy regimens [10]. The role of local therapies to the liver, such as liver resection, percutaneous ablative procedures, and liver-directed transarterial therapies, remains controversial as the presence of BCLM is generally seen as a disseminated disease requiring rather systemic chemotherapy than locoregional therapy.

However, in selected patients with unresectable and liver-dominant BCLM, palliation by transarterial therapies may be of interest. The following paragraphs provide an overview on the current results of TACE and TARE in patients with BCLM in metastatic breast cancer disease. To our knowledge, there are no data on TAE in patients with BCLM.

\section{TACE for BCLM}

Different TACE regimens for BCLM are described in the literature. A retrospective analysis by Giroux et al. [11] reported results of 8 patients who were treated by TACE due to pain $(n=4)$ and/or liver-dominant disease unresponsive to systemic chemotherapy. Only one patient suffered from extrahepatic disease, and 7 patients received systemic chemotherapy before TACE. Chemoembolization was performed with a mixture of $100 \mathrm{mg}$ cisplatin, $50 \mathrm{mg}$ doxorubicin, and $10 \mathrm{mg}$ of mitomycin mixed with ethiodized oil. Patients received a median of three TACE sessions. 5 patients (62.5\%) showed regression of liver tumors and one showed stable disease. 3 patients died within 3 months. The mean survival for the whole group was 20 months from the diagnosis of liver metastasis and 6 months from the first TACE.

A study by Li et al. [12] compared TACE and systemic chemotherapy in 48 patients with BCLM, of whom 20 were treated by systemic chemotherapy (cyclophosphamide, epirubicin, and 5-fluorouracil) and 28 by TACE. Patients were treated with 5 -fluorouracil and cisplatin followed by chemoembolization with a mixture of doxorubicin and iodized oil. The overall response rate was signifi- 
Table 2. Overview of studies using TARE in patients with BCLM

\begin{tabular}{|c|c|c|c|c|c|c|c|c|c|}
\hline Authors, year & $\begin{array}{l}\text { Number } \\
\text { of patients, } \\
n\end{array}$ & $\begin{array}{l}\text { Type of microspheres } \\
\text { (mean activity }^{\mathrm{a}} \text { ) }\end{array}$ & $\begin{array}{l}\text { Assessment } \\
\text { criteria }^{c}\end{array}$ & $\begin{array}{l}\text { Complete } \\
\text { response, } \\
\%\end{array}$ & $\begin{array}{l}\text { Partial } \\
\text { response, } \\
\%\end{array}$ & $\begin{array}{l}\text { Stable } \\
\text { disease, } \\
\%\end{array}$ & $\begin{array}{l}\text { Progressive } \\
\text { disease, } \\
\%\end{array}$ & $\begin{array}{l}\text { Median survival, } \\
\text { months }\end{array}$ & $\begin{array}{l}\text { Mean } \\
\text { survival, } \\
\text { months }\end{array}$ \\
\hline $\begin{array}{l}\text { Bangash et al., } \\
2007 \text { [34] }\end{array}$ & 27 & glass $(2.05 \pm 1.06 \mathrm{GBq})$ & WHO & 39 & 39 & 52 & 8.8 & $\mathrm{nr}$ & $\mathrm{nr}^{\mathrm{e}}$ \\
\hline $\begin{array}{c}\text { Jakobs et al., } \\
2008 \text { [17] }\end{array}$ & 30 & resin $(1.896 \mathrm{GBq})$ & RECIST & 0 & 61 & 35 & 4 & $\begin{array}{l}11.7 \text { (significantly } \\
\text { better for patients } \\
\text { with } C R \text { or } \mathrm{PR}^{\mathrm{d}} \text { ) }\end{array}$ & 9.6 \\
\hline $\begin{array}{l}\text { Haug et al., } \\
2012 \text { [35] }\end{array}$ & 58 & $\operatorname{resin}(1.77 \pm 0.49 \mathrm{GBq})$ & RECIST & 0 & 25.6 & 62.8 & 11.6 & 6.7 & $\mathrm{nr}$ \\
\hline $\begin{array}{l}\text { Gordon et al., } \\
2014 \text { [36] }\end{array}$ & 75 & glass (1.52 GBq) & RECIST & & 35.3 & 63.4 & 1.5 & 6.6 & $\mathrm{nr}$ \\
\hline $\begin{array}{c}\text { Saxena et al., } \\
2014 \text { [37] }\end{array}$ & 40 & $\operatorname{resin}(1.67 \pm 0.36 \mathrm{GBq})$ & RECIST & 5 & 26 & 39 & 29 & 13.6 & $\mathrm{nr}$ \\
\hline
\end{tabular}

${ }^{\mathrm{a} G l a s s}=$ Glass microspheres (TheraSphere; BTG International Ltd, London, UK). Resin = Resin microspheres (SIR-Spheres; SIRTex Medical Limited, Sydney, Australia).

${ }^{\mathrm{b}}$ Mean activity infused during TARE. Data are displayed in gigabecquerel (GBq).

${ }^{\mathrm{c}}$ Assessment criteria of response. WHO $=$ World Health Organization; RECIST $=$ response evaluation criteria in solid tumors.

${ }^{\mathrm{d}} \mathrm{CR}=$ Complete response; $\mathrm{PR}=$ partial response according to RECIST.

${ }^{\mathrm{e}} \mathrm{nr}=$ Not reported.

cantly better in patients treated by TACE (35.7\%) than in patients with systemic chemotherapy $(7.1 \%, \mathrm{p}<0.05)$. The $1-, 2-$, and 3 -year survival was better for TACE $(63.04,30.35$, and $13.01 \%$, respectively) than for systemic chemotherapy $(33.88,11.29$, and $0 \%$, respectively). However, 9 patients in the TACE group only had single liver metastasis and no extrahepatic disease, which might explain the good survival results. One patient died due to chronic renal and hepatic failure and one due to hepatic encephalopathy. Major limitations of the study were the lack of randomization and the fact that new systemic chemotherapies, e.g. with taxanes, were not tested against TACE.

The safety and efficacy of doxorubicin-loaded drug-eluting beads TACE (DEBDOX TACE) in patients with liver-dominant metastatic breast cancer was tested by Martin et al. [13]. The study population comprised 40 female patients with a total of 73 DEBDOX-TACE procedures. $57 \%$ of the patients had focal but stable extrahepatic disease (bone: $\mathrm{n}=16$; lung: $\mathrm{n}=3$; brain $\mathrm{n}=2$; pancreas $n=1) .62 \%$ of the patients had a tumor liver involvement of $<25 \%$; however, this was of a miliary and multifocal type. Concurrent systemic chemotherapy was present in the majority of patients. Adverse events were mostly limited to signs of a postembolization syndrome, except in one patient who required cholecystectomy following extrahepatic DC Beads infusion. The overall response rate at 3 months was $58 \%$ and then remained $50 \%$ at 6,9 , and 12 months following DEBDOX-TACE. The median progression-free survival (PFS) was 17 months, with a hepatic-specific PFS of 26 months. OS amounted to 47 months. Compared to first-line systemic chemotherapies with response rates of $30-65 \%$ and a PFS of 11 months [14], results of DEBDOX-TACE are encouraging.

A recently published phase II study with 43 patients analyzed TACE with gemcitabine in patients with BCLM [15]. The TACE regimen consisted of a mixture of gemcitabine $\left(1,200 \mathrm{mg} / \mathrm{m}^{2}\right)$, 100-300 mg degradable starch microspheres, and 2-10 ml of lipiodol. $88.4 \%$ of the patients received three cycles of TACE in a 4-week interval. 3 patients received only two cycles of intra-arterial gemcitabine due to tumor progression. Complete response (CR) and partial response (PR) was seen in $7 \%$, stable disease $(\mathrm{SD})$ in $39 \%$, and in $54 \%$ progressive disease was found. Median PFS was 3.3 months (95\% confidence interval (CI): 3.0-4.0). At 6 months, only $18 \%$ (95\% CI: 7-46\%) were free of progression. The estimated OS was 10.2 months (95\% CI: 18-59\%), and the estimated 1-year survival rate was $32 \%$.

Another study evaluated three different TACE regimens in a total of 208 patients [16]. TACE regimens consisted of mitomycinC only ( $\mathrm{n}=76)$, mitomycin-C with gemcitabine $(\mathrm{n}=111)$, and gemcitabine only $(n=21)$. Embolization was performed by mixing the chemotherapeutic agent(s) with lipiodol, followed by injection of 200-450 mg of starch microspheres (200-450 $\mu \mathrm{m})$. TACE was performed at 4-week intervals (mean: 5.1 sessions; range: 3-25 sessions). Of the three treatment regimens, the combination of mitomycin-C and gemcitabine performed best with PR in $17.12 \%$, SD in $49.55 \%$, and progressive disease in $33.33 \%$. Median and mean survival times were best after TACE with a combination of mitomycin-C and gemcitabine, i.e. 24.8 and 35.5 months, respectively.

\section{TARE for BCLM}

There are only a few studies available which exclusively report on TARE in patients with BCLM. Table 2 provides an overview on these studies with regard to response rate, survival, and toxicity of TARE in patients with BCLM.

Disease control rates, defined as the sum of CR, PR, and SD, varied from 70 to $98.7 \%$ in the mentioned studies. These results are 
actually very encouraging, considering the fact that the included patients suffered from advanced disease especially in the liver and had several chemotherapies. Jakobs et al. [17] reported a median OS of 11.7 months (range: 3.0-45.1 months). Patients who responded to TARE showed a significantly better survival than those who did not respond ( 23.6 vs. 5.7 months, $\mathrm{p}=0.005$ ). There was no significant difference in survival for patients with and without extrahepatic disease.

\section{Neuroendocrine Tumor Liver Metastases}

\section{TAE for NET}

TAE for NET might be indicated in patients with defined lesions and low proliferation rates as well as in functional tumors. The first publication of TAE in patients with liver metastases of NET was by Carrasco et al. [18] where a response to TAE was observed in over $90 \%$ of the patients, with a mean response of 11 months. High tumor response rates with regard to radiological changes as well as biochemical response were subsequently published in many case series, and the median OS differed from 10 to 80 months [19]. In a large study in 107 patients with carcinoid syndrome, a reduction of associated symptoms was described in $80 \%$ of the patients [20]. Further studies considering the safety of TAE in patients with hepatic metastases of NET reported rare adverse events such as ischemia of the biliary tree and postembolization syndrome. In the latter review, complications were seen in 125/896 (14\%) [19]. Overall, TAE seems to be an effective and safe procedure in patients with small to moderate tumor involvement in the liver and non-compromised liver function. Patients with carcinoid symptoms and associated cardiac disease must be treated with specific caution since treatment-related mortality might be higher due to hormonal release.

\section{TACE for NET}

In contrast to TAE, TACE does rely on the combination of chemotherapy and embolization of liver metastases. The most commonly used chemotherapies are anthracyclines such as doxorubicin, combinations of cisplatin, doxorubicin, and mitomycin-C, or streptozotocin. Despite the moderate activity of chemotherapy especially in low proliferative NET, TACE in NET has high activity since the concentration of the cytotoxic agent in combination with embolization is 100 -fold [21]. When looking at the evidence, however, there are just a few trials focusing on the isolated use of TACE and not TAE. Pitt et al. [22] compared the outcomes of TACE versus TAE in 100 patients with carcinoid or islet cell tumors. TACE was performed using cisplatin, doxorubicin, and mitomycin-C. No differences were found regarding OS, response rates, and 5-year survival. There were no differences in complications (2.4 vs. $6.6 \%$ ) and mortality rates $(0.8$ vs. $1.8 \%)$ in this trial. A large study by Bloomston et al. [23] analyzed 122 patients with metastatic carcinoid tumors undergoing TACE with a median follow-up of 21.5 months. Complications occurred in a large fraction of $23 \%$, and periprocedural mortality was $5 \%$. This study concludes that TACE offers good symptom palliation and long-term survival. However, it should be used cautiously in patients with large tumor burden. A recent trial using drug-eluting beads (DEB-TACE) in patients with liver metastases of NET showed a reduction of the targeted lesion by $78 \%$ when taking criteria of the European Association for the Study of the Liver into account. However, $54 \%$ of the patients were developing biloma as a substantial side effect [24].

\section{TARE for NET}

In contrast to TAE and TACE, TARE is not embolizing the tumor lesions and is therefore generally well tolerated even in higher tumor burden. Thus, the major aim of TARE is not to compete with TACE or TARE but to treat different situations with large tumors since it is generally, if well used, a more secure treatment with less side effects. Many phase II studies have already been published. The largest series by Kennedy [25] showed a PR in 61\% of the patients with low toxicities, mainly fatigue (6.5\%). A recent study by Peker et al. [26] demonstrated the safety and effectiveness of the treatment of unresectable liver NET, with 1- and 2-year survival rates of 71 and $45 \%$, respectively. A recent meta-analysis by Devcic et al. [27] showed an average objective response of $50 \%$ and a weighted average disease control rate of $86 \%$. This large metaanalysis considered TARE as an effective treatment option for patients with hepatic metastatic NET with high response rates and prolonged survival.

Some experts still consider TARE in NET as an experimental procedure. However, in the light of current data and its relation to existing data for TACE and TAE, we strongly suggest to regard TARE not as competition to existing therapies but as a safe and effective complementation especially in larger tumor burden.

\section{Melanoma Liver Metastases}

\section{TAE/TACE for Melanoma Liver Metastases}

To our knowledge, there is no data for bland embolization in melanoma liver metastases. Some studies have been published on hepatic intra-arterial chemotherapy (HIAC) and TACE in melanoma liver metastases. Most of the HIAC trials were retrospective or phase II trials by Becker et al. [28] combining fotemustine with sequential IFNa + IL-2; here, an overall response of $22 \%$ with a median OS of 12.1 months was revealed. A larger phase III trial in ocular melanoma in 171 patients was only published as an abstract and showed an overall response of $11 \%$ and an OS of 14.6 months [29]. TACE using various chemotherapies such as cisplatin, paclitaxel, fotemustine, doxorubicin, or even immunogenic agents such as GM-GSF resulted in response rates of up to $39 \%$ in a retrospective series combining TACE with HIAC [30]. However, the patient series remained small and, to our knowledge, there is no phase III trial focusing on the efficacy of TACE in this patient population.

\section{Chemosaturation for Melanoma Liver Metastases}

Chemosaturation isolates the hepatic perfusion, thus establishing high doses in the liver. The agent commonly used is melphalan. 
A large retrospective study with 125 patients by Gupta et al. [31] led to $11 \% \mathrm{PR}, 16 \%$ minor responses, an SD of $65 \%$, and progressive disease in $8 \%$. However, this is a complicated procedure with long procedure times of up to $8 \mathrm{~h}$, considerable morbidity, and prolonged hospital stay of up to 10 days [32].

\section{TARE for Melanoma Liver Metastases}

A recent study by Xing et al. [33] analyzed the efficacy of TARE compared to best supportive care in 58 patients with liver metastases of melanoma. Both groups were well balanced. The median OS was highly significantly better in the treatment group (19.9 vs. 4.8 months, $\mathrm{p}<0.0001$ ). Toxicity was rather low with abdominal pain in $17.9 \%$, fatigue in $14.3 \%$, and self-limiting grade III bilirubin toxicity in $10.7 \%$.

\section{Conclusion}

In conclusion, intra-arterial therapies in the form of TAE, TACE, or TARE are secure and highly effective. Each of these different modalities must be chosen in regard to available data but also according to personalized patient level. However, more standardized treatment protocols and phase III data are desperately needed to establish transparent and useful guidelines.

\section{Disclosure Statement}

We disclose no financial support or author involvement with organization(s) with financial interest in the subject matter.

\section{References}

1 Murata S, Iwahashi K, Yasunaga T, Hasegawa T, Matsushita S: Intraarterial infusion of anti-cancer agents. (A suitable technic introducing a polyethylene tube, with angiographic visualization, through the deep femoral artery directly into the artery in the abdomen selected for injection, using two kinds of guidewires) (author's transl) (Article in Japanese). Nihon Gan Chiryo Gakkai Shi 1976;11:227-239.

2 Ariel IM, Padula G: Treatment of symptomatic metastatic cancer to the liver from primary colon and rectal cancer by the intraarterial administration of chemotherapy and radioactive isotopes. J Surg Oncol 1978; 10:327-336.

3 Lammer J, Malagari K, Vogl T, et al.; PRECISION V Investigators: Prospective randomized study of doxorubicin-eluting-bead embolization in the treatment of hepatocellular carcinoma: results of the PRECISION V study. Cardiovasc Intervent Radiol 2010;33:41-52.

4 Hong K, Khwaja A, Liapi E, Torbenson MS, Georgiades CS, Geschwind JF: New intra-arterial drug delivery system for the treatment of liver cancer: preclinical assessment in a rabbit model of liver cancer. Clin Cancer Res 2006;12:2563-2567.

5 Brown DB, Nikolic B, Covey AM, Nutting CW, Saad WE, Salem R, Sofocleous CT, Sze DY; Society of Interventional Radiology Standards of Practice Committee: Quality improvement guidelines for transhepatic arterial chemoembolization, embolization, and chemotherapeutic infusion for hepatic malignancy. J Vasc Interv Radiol 2012;23:287-294.

-6 Berger DH, Carrasco CH, Hohn DC, Curley SA: Hepatic artery chemoembolization or embolization for primary and metastatic liver tumors: post-treatment management and complications. J Surg Oncol 1995;60: 116-121.

7 Basile A, Carrafiello G, Ierardi AM, Tsetis D, Brountzos E: Quality-improvement guidelines for hepatic transarterial chemoembolization. Cardiovasc Intervent Radiol 2012;35:765-774.

$>$ Koops A, Wojciechowski B, Broering DC, Adam G, Krupski-Berdien G: Anatomic variations of the hepatic arteries in 604 selective celiac and superior mesenteric angiographies. Surg Radiol Anat 2004;26:239-244.

9 Morsbach F, Sah BR, Spring L, Puippe G, Gordic S, Seifert B, Schaefer N, Pfammatter T, Alkadhi H, Reiner CS: Perfusion CT best predicts outcome after radioembolization of liver metastases: a comparison of radionuclide and CT imaging techniques. Eur Radiol 2014; 24:1455-1465.
10 Pentheroudakis G, Fountzilas G, Bafaloukos D, Koutsoukou V, Pectasides D, Skarlos D, Samantas E, Kalofonos HP, Gogas H, Pavlidis N: Metastatic breast cancer with liver metastases: a registry analysis of clinicopathologic, management and outcome characteristics of 500 women. Breast Cancer Res Treat 2006;97:237-244.

11 Giroux MF, Baum RA, Soulen MC: Chemoembolization of liver metastasis from breast carcinoma. J Vasc Interv Radiol 2004;15:289-291.

12 Li XP, Meng ZQ, Guo WJ, Li J: Treatment for liver metastases from breast cancer: results and prognostic factors. World J Gastroenterol 2005;11:3782-3787.

13 Martin RC, Robbins K, Fages JF, Romero FD, Rustein L, Tomalty D, Monaco R: Optimal outcomes for liverdominant metastatic breast cancer with transarterial chemoembolization with drug-eluting beads loaded with doxorubicin. Breast Cancer Res Treat 2012;132: $753-763$.

14 Beslija S, Bonneterre J, Burstein HJ, et al.; Central European Cooperative Oncology Group (CECOG): Third consensus on medical treatment of metastatic breast cancer. Ann Oncol 2009;20:1771-1785.

15 Eichler K, Jakobi S, Gruber-Rouh T, Hammerstingl R, Vogl TJ, Zangos S: Transarterial chemoembolisation (TACE) with gemcitabine: phase II study in patients with liver metastases of breast cancer. Eur J Radiol 2013;82:e816-822.

16 Vogl TJ, Naguib NN, Nour-Eldin NE, Eichler K, Zangos S, Gruber-Rouh T: Transarterial chemoembolization (TACE) with mitomycin $\mathrm{C}$ and gemcitabine for liver metastases in breast cancer. Eur Radiol 2010;20: $173-180$.

17 Jakobs TF, Hoffmann RT, Fischer T, Stemmler HJ, Tatsch K, La Fougere C, Murthy R, Reiser MF, Helmberger TK: Radioembolization in patients with hepatic metastases from breast cancer. J Vasc Interv Radiol 2008;19:683-690.

18 Carrasco CH, Charnsangavej C, Ajani J, Samaan NA, Richli W, Wallace S: The carcinoid syndrome: palliation by hepatic artery embolization. AJR Am J Roentgenol 1986;147:149-154.

19 Del Prete M, Fiore F, Modica R, Marotta V, Marciello F, Ramundo V, Di Sarno A, Carratu A, di Roseto Cde L, Tafuto S, Tatangelo F, Baldelli R, Colao A, Faggiano A; Multidisciplinary Group for NeuroEndocrine Tumors of Naples: Hepatic arterial embolization in patients with neuroendocrine tumors. J Exp Clin Cancer Res 2014;33:43
20 Ho AS, Picus J, Darcy MD, Tan B, Gould JE, Pilgram TK, Brown DB: Long-term outcome after chemoembolization and embolization of hepatic metastatic lesions from neuroendocrine tumors. AJR Am J Roentgenol 2007;188:1201-1207.

21 Konno T: Targeting chemotherapy for hepatoma: arterial administration of anticancer drugs dissolved in $\mathrm{Li}^{-}$ piodol. Eur J Cancer 1992;28:403-409.

22 Pitt SC, Knuth J, Keily JM, McDermott JC, Weber SM, Chen H, Rilling WS, Quebbeman EJ, Agarwal DM, Pitt HA: Hepatic neuroendocrine metastases: chemo- or bland embolization? J Gastrointest Surg 2008;12:19511960

23 Bloomston M, Al-Saif O, Klemanski D, Pinzone JJ, Martin EW, Palmer B, Guy G, Khabiri H, Ellison EC, Shah MH: Hepatic artery chemoembolization in 122 patients with metastatic carcinoid tumor: lessons learned. J Gastrointest Surg 2007;11:264-271.

24 Bhagat N, Reyes DK, Lin M, Kamel I, Pawlik TM, Frangakis C, Geschwind JF: Phase II study of chemoembolization with drug-eluting beads in patients with hepatic neuroendocrine metastases: high incidence of biliary injury. Cardiovasc Intervent Radiol 2013;36: 449-459.

25 Kennedy AS, Dezarn WA, McNeillie P, et al: Radioembolization for unresectable neuroendocrine hepatic metastases using resin 90Y-microspheres: early results in 148 patients. Am J Clin Oncol 2008;31:271-279.

26 Peker A, Çiçek O, Soydal Ç, Küçük NÖ, Bilgiç S: Radioembolization with yttrium-90 resin microspheres for neuroendocrine tumor liver metastases. Diagn Interv Radiol 2015;21:54-59.

27 Devcic Z, Rosenberg J, Braat AJ, Techasith T, Banerjee A, Sze DY, Lam MG: The efficacy of hepatic 90 Y resin radioembolization for metastatic neuroendocrine tumors: a meta-analysis. J Nucl Med 2014;55:1404-1410.

28 Becker JC, Terheyden P, Kämpgen E, Wagner S, Neumann C, Schadendorf D, Steinmann A, Wittenberg G, Lieb W, Bröcker EB: Treatment of disseminated ocular melanoma with sequential fotemustine, interferon alpha, and interleukin 2. Br J Cancer 2002;87:840-845.

29 Leyvraz S, Suciu S, Piperno-Neumann S, et al: Randomized phase III trial of intravenous (IV) versus hepatic intra-arterial (HIA) fotemustine in patients with liver metastases from uveal melanoma: final results of the EORTC 18021 study. J Clin Oncol 2012;30(suppl): abstr 8532 . 
30 Ahrar J, Gupta S, Ensor J, et al: Response, survival, and prognostic factors after hepatic arterial chemoembolization in patients with liver metastases from cutaneous melanoma. Cancer Invest 2011;29:49-55.

31 Gupta S, Bedikian AY, Ahrar J, et al: Hepatic artery chemoembolization in patients with ocular melanoma metastatic to the liver: response, survival, and prognostic factors. Am J Clin Oncol 2010;33:474-480.

32 Alexander HR, Libutti SK, Pingpank JF, et al: Hyperthermic isolated hepatic perfusion using melphalan for patients with ocular melanoma metastatic to liver. Clin Cancer Res 2003;9:6343-6349.
3 Xing M, Prajapati HJ, Dhanasekaran R, et al: Selective internal yttrium-90 radioembolization therapy $(90 \mathrm{Y}-$ SIRT) versus best supportive care in patients with unresectable metastatic melanoma to the liver refractory to systemic therapy: safety and efficacy cohort study. Am J Clin Oncol 2014;DOI: 10.1097/COC.0000000000000109.

34 Bangash AK, Atassi B, Kaklamani V, et al: 90Y radioembolization of metastatic breast cancer to the liver: toxicity, imaging response, survival. J Vasc Interv Radiol 2007; 18:621-628.

5 Haug AR, Teiga Donfack BP, Trumm C, et al: 18FFDG PET/CT predicts survival after radioembolization of hepatic metastases from breast cancer. J Nucl Med 2012;53:371-377
36 Gordon AC, Gradishar WJ, Kaklamani VT, et al: Yttrium-90 radioembolization stops progression of targeted breast cancer liver metastases after failed chemotherapy. J Vasc Interv Radiol 2014;25:1523-1532, 1532.el-2.

37 Saxena A, Kapoor J, Meteling B, et al: Yttrium-90 radioembolization for unresectable, chemoresistant breast cancer liver metastases: a large single-center experience of 40 patients. Ann Surg Oncol 2014;21:12961303. 